\title{
Mode multiplexing and holographic demultiplexing communication channels on a multimode fiber
}

\author{
Mark Saffman and Dana Z. Anderson \\ Department of Physics and Joint Institute for Laboratory Astrophysics, University of Colorado, Boulder, Colorado 80309-0440
}

Received August 22, 1990; accepted December 18, 1990

\begin{abstract}
Spatial mode multiplexing is used to transmit several communication channels on a single multimode optical fiber. Each channel is encoded by an orthogonal pattern produced by a spatial light modulator. A photorefractive medium holographically decodes the output speckle pattern at a receiver station. We demonstrate ring and star architectures for interconnection networks. Typical cross-talk-to-signal ratios, for fully interconnected threeprocessor networks, are -24 and $-26 \mathrm{~dB}$ for the ring and star, respectively.
\end{abstract}

A multimode fiber illuminated with light from a monochromatic source produces a complicated speckle pattern that arises from the interference of many fiber modes. ${ }^{1}$ It is difficult if not impossible to say specifically what the output pattern will be, especially if the number of propagating modes is large. Suppose that the fiber is illuminated with two different optical fields that are electric-field orthogonal at some input reference plane. Even if their spatial distribution is not known, the two output patterns are guaranteed to be field-amplitude orthogonal, provided that there is no light lost between the input plane and the output. The orthogonality is guaranteed by the unitarity of the fiber, viewed mathematically as an energy-preserving linear transformation operator. ${ }^{2}$ Even if there is modest loss, in many cases the outputs will be orthogonal, or nearly so.

Two speckle patterns produced by sources at different locations along a single fiber are not guaranteed to be orthogonal. The expected value of the overlap of two randomly chosen equal-intensity speckle patterns of an $N$ mode fiber is $1 / N$. For a large $N$, the probability that the overlap deviates from its expected value drops off so sharply with the deviation that for some practical purposes the patterns may be taken to be orthogonal. For example, for a fiber carrying 10,000 modes and 100 random patterns, the probability that any two patterns have an overlap integral greater than 0.1 is $P<10^{-220 \text { ! }}$

In this Letter we demonstrate a technique for signal multiplexing on a multimode fiber ${ }^{3,4}$ that is based on the unitarity principle and capitalizes on the high dimensionality of speckle patterns. Time signals are encoded by their spatial amplitude and phase pattern at the transmitter. They are decoded by a dynamic volume hologram, ${ }^{5}$ which is formed by interfering the output speckle pattern with itself at the receiver. ${ }^{6}$ Speckle patterns derived from input patterns orthogonal to the ones used to write the hologram will not give rise to a strong reconstructed object beam. That the hologram is dynamic ameliorates the obvious problem of fiber movement, for if it moves a substantial amount, the speckle pattern for a given input changes, but the hologram can be refreshed. It also permits the interconnects between a collection of transmitters and receivers to be reconfigurable. ${ }^{7}$

The principle of the technique is easily demonstrated by injecting the light transmitted by a mask with a rectangular hole into a multimode fiber, as shown in Fig. 1. A volume hologram is made with this light, then, as the mask is translated, the holographically diffracted light intensity will decrease, as illustrated in Fig. 2. Ideally, when the mask has been translated a distance greater than its width, the diffracted intensity should drop to zero. Similar results were obtained with a 70-m length of fiber wrapped on a 15-cm-diameter spool and having $\sim 50 \%$ transmission loss, although the background signal, with the mask completely displaced, was approximately twice as high. We have also observed the same behavior when varying the electric-field polarization angle: rotating the polarization by $\theta$ from that used to write the hologram causes the diffracted light to decrease as $\cos ^{2}(\theta)$.

Figure 3 shows that the discrimination against orthogonal spatial patterns improves as the grating strength increases, up to the point where beam fanning degrades the results. However, a signal-to-crosstalk ratio of better than $50: 1$, or $34 \mathrm{~dB}$ after detection, can be achieved for $45 \%$ diffraction efficiency gratings.

We have constructed ring and star interconnection networks, each having three transmitter $(\mathrm{T}) /$ receiver

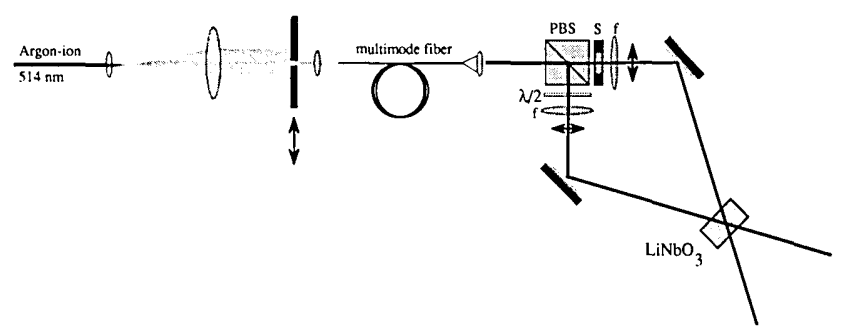

Fig. 1. Mode multiplexing in a multimode fiber. The fiber is step index, with a $100-\mu \mathrm{m}$ core and a $50-\mathrm{cm}$ length. The $\mathrm{LiNbO}_{3}$ crystal is $6 \mathrm{~mm}$ thick and doped with $0.015 \mathrm{wt}$. \% Fe, and the beam overlap region is approximately $0.2 \mathrm{~mm} \times 0.2$ $\mathrm{mm} \times 1.1 \mathrm{~mm}$. The receiver is composed of a polarizing beam splitter (PBS), a shutter (S), and 20 - and $40-\mathrm{mm}$ focallength lenses $(\mathrm{f})$. 


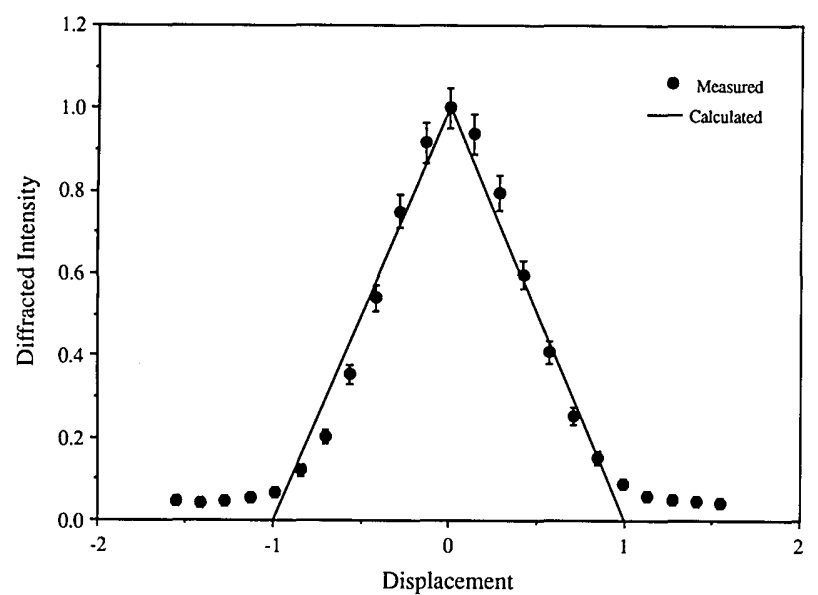

Fig. 2. Variation of diffracted signal with the input pattern. The calculated lines correspond to the area overlap of two displaced rectangles and therefore do not account for diffraction from the edges.

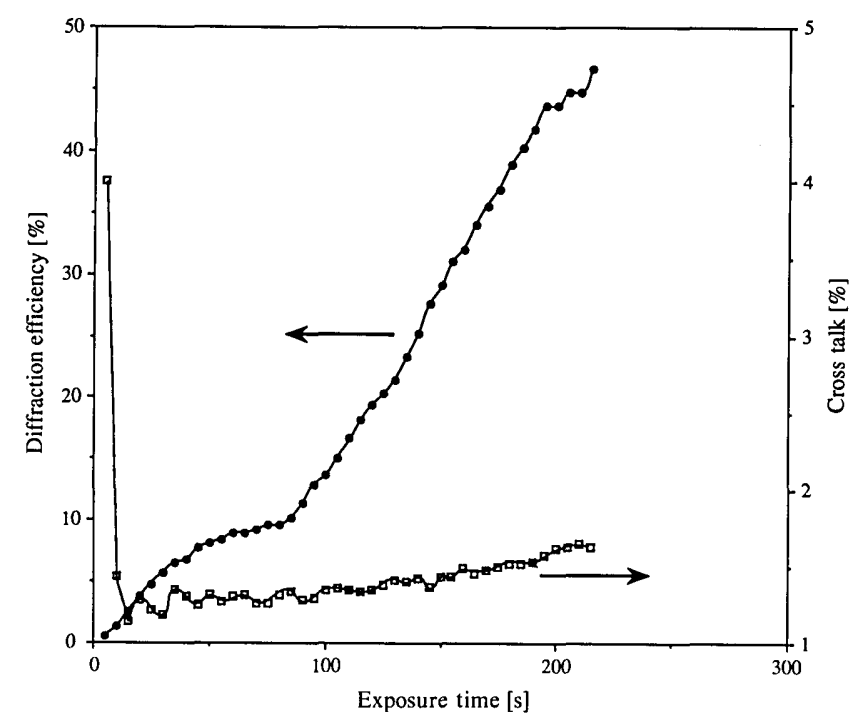

Fig. 3. Diffraction efficiency and cross talk versus exposure time for a single hologram. Cross talk is defined as the diffracted signal from the orthogonal input pattern divided by the diffracted signal from the original input pattern. The writing intensity is $0.7 \mathrm{~W} / \mathrm{cm}^{2}$.

(R) nodes, as shown in Fig. 4. Each transmitter has an acousto-optic modulator to produce an intensity-modulated signal and a spatial light modulator to encode the signal on a given channel. In our case, the light modulator is simply a piece of glass mounted at an oblique angle to the beam, which, when rotated, selects an input angle to the fiber. The beam numerical aperture was $\sim 0.02$, compared with 0.3 for the stepindex fiber. The receiver at each node of the ring network uses a polarizing beam splitter to separate the polarization components of the speckle pattern, thereby producing two speckle patterns. The interference pattern produced over the entire intersection of the two speckle patterns is recorded in a photorefractive crystal. The star network uses a single photorefractive crystal to perform the interconnects, as shown in
Fig. 4(b). Normally the signals would be coupled back into multimode fibers for propagation to the receivers; in the experiment we simply have detectors at the output from the hologram.

The ring and star architectures are in many ways complementary. For a fully interconnected $N$-node network the ring requires the storing of $O(N)$ holograms in $N$ separate crystals, whereas the star stores $O\left(N^{2}\right)$ holograms in a single crystal. The star places higher demands on the holographic medium but eliminates the loss at the passive node couplers, ${ }^{8}$ inherent to the ring architecture. In the ring architecture the transmitters may be mutually incoherent, but in the star the interconnects are formed by the interference of patterns from separate nodes, so all transmitters must be mutually coherent. In both structures the switching speed is limited by the spatial light modulator response time, once the interconnection holograms have been recorded.

The ring network interconnections were written for each node sequentially. For example, two 5\% efficiency holograms were recorded at node 1 by sequentially

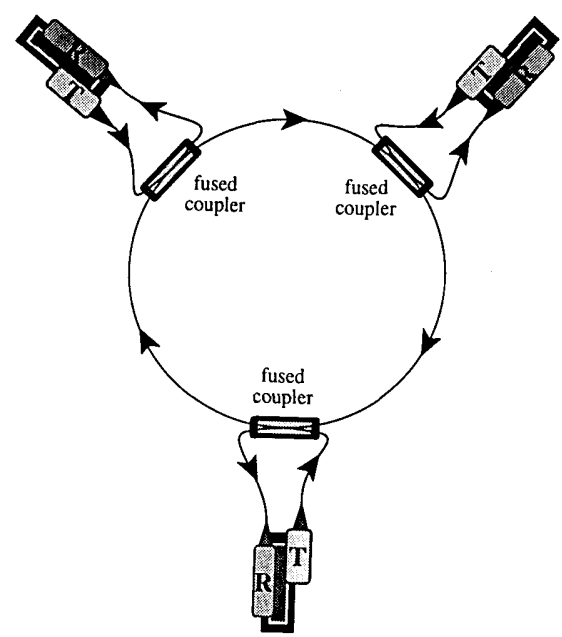

(a)

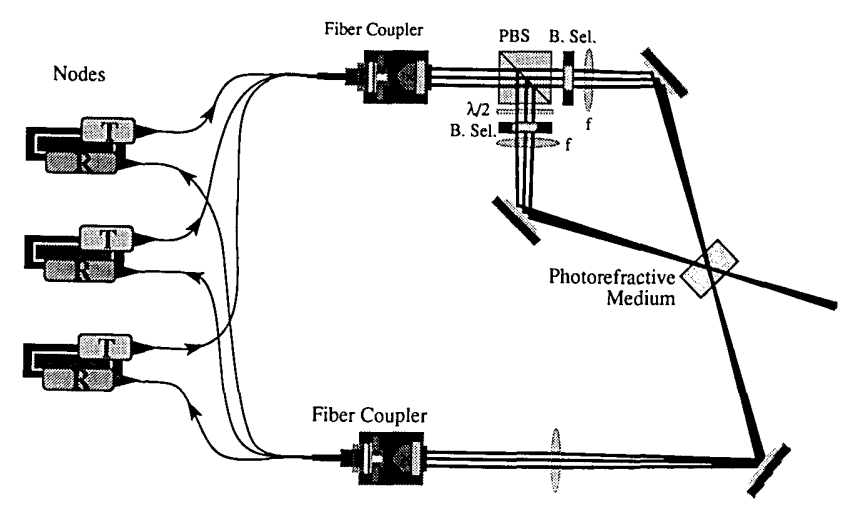

(b)

Fig. 4. Ring and star optical interconnection networks. (a) The ring nodes are connected to the network with symmetric multimode couplers. (b) The star uses a single interconnection crystal as an optical crossbar. The fiber coupler is a $10 \times$ microscope objective, and the beam overlap region is approximately $0.9 \mathrm{~mm} \times 0.9 \mathrm{~mm} \times 5.1 \mathrm{~mm}$. B. Sel., beam selector; f's, 40-mm focal-length lenses. 


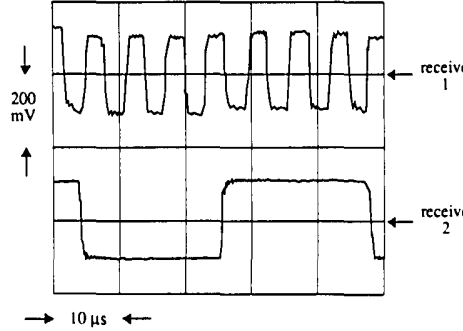

(a)

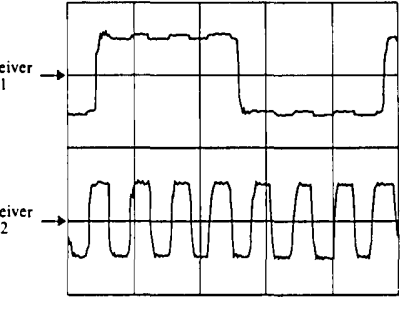

(b)
Fig. 5. Data switching in the star network with two simultaneous talkers. Nodes 1 and 2 transmit at 23 and $160 \mathrm{kHz}$. In (a) 1 talks to 2 and 2 talks to 1 ; in (b) 1 talks to 1 and 2 talks to 2 .

transmitting pattern 1 from nodes 2 and 3 . The worst-case cross talk, defined as the diffracted signal at a node relative to the undesired signal due to the same node's simultaneously transmitting, was measured at each node. Typical cross-talk levels were -25 and $-22 \mathrm{~dB}$, after detection, for transmission to the nearest and next-nearest neighbor nodes, respectively.

The star network was initialized by having pairs of nodes transmit simultaneously. The signals from the intended transmitter and receiver nodes were then selected as reference and object beams, respectively. To interconnect the three-node network fully the exposure times were varied from 10.6 to $6 \mathrm{~s}$ (Ref. 9) to write six equal-strength holograms of $~ 5 \%$ diffraction efficiency. The typical switching performance with two simultaneous talkers is shown in Fig. 5. Crosstalk levels were measured for each set of interconnections. Owing to small variations in the strength of the holograms, the spread was from -24 to $-27 \mathrm{~dB}$, with an average of $-26 \mathrm{~dB}$.

In a communication system based on this technique we have two choices for the laser source: the entire network can be supplied by a single high-power source (as we have done), or each transmitter can have its own source. In the case of a single source we are free to supply a nice object beam, which would be ideal from a holography standpoint. Recording the interference between two speckle patterns is in many respects an odd thing to do, but it does permit, for example, the source lasers to be mutually incoherent.

Under the proper circumstances the volume hologram can provide a measure of the amplitude overlap, that is, the dot product between a write and a read beam along one spatial dimension ${ }^{10}$; ideally, one would use a waveguide that is single mode in one dimension and multimode in the other. In our configuration then, the effective number of modes is the square root of the number of modes (per polarization direction) of the two-dimensional fiber. Even in the one-dimensional case, we know little about the properties of superimposed holograms written with pairs of speckle patterns. ${ }^{11}$ We do know, for example, that a single such hologram is not linear in the product of the fields because both beams are amplitude modulated and therefore the recording medium time constant will be space dependent. In any case, we expect that it is the holographic aspects that will limit the channel-carrying capacity of the scheme, not the fiber itself.

In any practical version of these networks the dynamic recording medium needs to be periodically updated. The holograms erase while being used, and any mechanical perturbations to the fibers cause fluctuations in the mode patterns, which leads to reduced diffraction efficiency and increased cross talk. We note that the ring architecture is relatively insensitive to fiber movement since each hologram is derived from a single speckle pattern. To avoid dead time, we envisage a twin-bus structure that uses one bus while the holographic media of the other are refreshed. It may be helpful in a practical device to employ a high-speed photorefractive material such as $\mathrm{Bi}_{12} \mathrm{SiO}_{20}$ or GaAs.

The authors are grateful to C. Benkert for fruitful discussions. This research was supported by U.S. Air Force Office of Scientific Research grant AFOSR-900198. Mark Saffman acknowledges support provided by a fellowship from the National Science Foundation Engineering Research Center in Optoelectronic Computing Systems at the University of Colorado and a U.S. Air Force Office of Scientific Research laboratory graduate fellowship. Dana Z. Anderson is an Alfred P. Sloan Research Fellow.

\section{References}

1. For an interesting discussion on fiber modes, see A. Yariv, J. Opt. Soc. Am. 66, 301 (1976).

2. On unitarity see, for example, L. D. Landau and E. M. Lifshitz, Quantum Mechanics, Non-Relativistic Theory, 2nd ed. (Pergamon, Oxford, 1965), p. 35.

3. For a description of other fiber-multiplexing techniques, see A. B. Sharma, S. J. Halme, and M. M. Butusov, Optical Fiber Systems and Their Components (Springer-Verlag, Berlin, 1981).

4. Related research on spatial mode multiplexing, which was, however, limited to fibers without mode coupling, has been reported by S. Berdague and P. Facq, Appl. Opt. 21, 1950 (1982).

5. L. Solymar and D. J. Cooke, Volume Holography and Volume Gratings (Academic, London, 1981).

6. Holographic detection for fiber sensors has been used by T. J. Hall, M. A. Fiddy, and M. S. Ner, Opt. Lett. 5, 485 (1980), and G. Indebetouw, K. D. Bennett, P. Zhang, and R. G. May, IEEE J. Lightwave Technol. 8, 1039 (1990).

7. For a recent review of the field see J. W. Goodman, in Optical Processing and Computing, H. H. Arsenault, T. Szoplik, and B. Macukow, eds. (Academic, Boston, Mass., 1989), Chap. 1.

8. The fused coupler losses are approximately mode independent, so field orthogonality, but not unitarity, is preserved.

9. K. Blфtekjær, Appl. Opt. 18, 57 (1979).

10. D. Z. Anderson and D. M. Lininger, Appl. Opt. 26, 5031 (1987).

11. B. Y. Zel'dovich, V. V. Shkunov, and T. V. Yakovleva, Sov. Phys. Usp. 29, 678 (1986). 